\title{
Construcción de una escala de habilidades parentales en madres y padres
}

Construction of the of parenting skills scale, in both parents

\author{
Gustavo Martínez Guadarrama \\ Erika Robles Estrada \\ Johannes Oudhof Van Barneveld \\ Susana Silvia Zarza Villegas \\ Lourdes Gabriela Villafaña Montiel \\ Universidad Autónoma del Estado de México
}

\section{Resumen}

El objetivo de esta investigación fue construir una escala válida y confiable que midiera las habilidades parentales en padres y madres. Se trabajó con 341 hombres y 341 mujeres de la ciudad de Toluca con hijos entre 6 a 12 años de edad. Con base en las siguientes habilidades: educativas, de agencia parental, de autonomía y apoyo social, vida personal y de pareja, y organización doméstica (Rodrigo \& Máiquez, 2008). El procedimiento que se llevó a cabo fue someter el instrumento preliminar a validez de jueces; posteriormente, se llevó a cabo un pilotaje y la aplicación final del instrumento de forma colectiva. El procesamiento de la información para la validez se realizó mediante un análisis factorial exploratorio de tipo ortogonal con rotación varimax $y$, para la confiabilidad, el alpha de Cronbach. De acuerdo con los resultados, en el instrumento de hombres se obtuvieron 8 dimensiones; en el instrumento de mujeres se encontraron 5. En ambos padres se encontraron habilidades de afecto, acuerdos en la pareja, limpieza y seguridad, estimulación y desarrollo y autoeficacia parental. Los padres presentaron tres más: afecto, solución de problemas y adaptación a las características del menor. Ambas escalas son válidas y confiables, de tal manera que se concluye que ambos padres consideran importantes todas las habilidades en la crianza cotidiana, las cuales son esenciales para el desarrollo biopsicosocial de los niños.

Palabras Clave: habilidades parentales, validez, confiabilidad, padres, madres.

Nota de Autor:

Gustavo Martínez Guadarrama, Facultad de Ciencias de la Conducta, Universidad Autónoma del Estado de México; Erika Robles Estrada, Facultad de Ciencias de la Conducta, Universidad Autónoma del Estado de México; Johannes Oudhof Van Barneveld, Facultad de Ciencias de la Conducta, Universidad Autónoma del Estado de México; Susana Silvia Zarza Villegas, Facultad de Ciencias de la Conducta, Universidad Autónoma del Estado de México; Lourdes Gabriel Villafaña Montiel, Facultad de Ciencias de la Conducta, Universidad Autónoma del Estado de México.

La correspondencia en relación con este artículo debe dirigirse a Gustavo Martínez Guadarrama, Facultad de Ciencias de la Conducta, Universidad Autónoma del Estado de México, Carretera Toluca-Naucalpan km. 1.5 s/n, Toluca, México, C.P. 50010

Correo electrónico: buztavo@ hotmail.com 


\begin{abstract}
The objective of this research was to construct a valid and reliable scale to measure parenting skills in parents. We worked with 341 men and 341 women from Toluca city whose children were between 6-12 years of age. The skills relied on: educational, parental agency, autonomy and social support, personal life and relationship, and domestic organization (Rodrigo \& Máiquez, 2008). The procedure carried out was: first to submit to judges' preliminary instrument validity, then a pilot was conducted and finally, the application of the instrument collectively. The information processing is carried out for validity using exploratory factor analysis of orthogonal type with varimax rotation and reliability Cronbach's alpha. According to the results, eight dimensions were obtained by the men's instrument and five dimensions were obtained by the women's instrument. In both, parents affection, partner agreements, cleaning and security, stimulation and development and parental self-efficacy skills were found. Male parents showed three more: affection, problem solving and adaptation to the characteristics of the child. Both scales are valid and reliable, so that it is concluded that both parents considered important all everyday parenting skills which are essential for the biopsychosocial development of children.
\end{abstract}

Keywords: parenting skills, validity, reliability, fathers, mothers.

En la vida del ser humano, la existencia de una familia es una necesidad básica para la sobrevivencia y el sano desarrollo físico y psicológico de niños y adolescentes. De hecho, es la principal responsable de llevar a cabo la socialización primaria y la integración de los menores al ámbito sociocultural. Para Musitu y Cava (2001), la socialización es un proceso vitalicio y bidireccional mediante el cual las personas adquieren los valores, creencias, normas y formas de conducta apropiados para la sociedad a la que pertenecen. Es decir padres e hijos aprenden mutuamente el cómo ejercer su papel en la vida cotidiana. El niño asimila conocimientos, actitudes, valores, costumbres, sentimientos y demás patrones culturales. A los padres se les considera como las personas conocedoras de estos elementos culturales que el niño desconoce, además de ser poseedores de un desarrollo cognitivo, social y afectivo del cual carece el niño, así como de un alto grado de poder (físico, derivado de sus mayores conocimientos y experiencia, económico, etc. [Musitu \& García, 2001]).
La socialización se manifiesta en gran medida en las prácticas parentales, o bien en lo que hacen (o no hacen) los padres en la vida cotidiana para educar a sus hijos. Moral (2013), enfatiza la importancia de estas prácticas al señalar que se centran en las conductas específicas que realizan madres y padres para lograr sus objetivos respecto a la crianza y su relación con el cuidado, protección, la enseñanza y la expresión de amor hacia los hijos. Estos elementos señalados por el autor son indicativos de que la figura de ambos padres necesita disponer de ciertas competencias que permitan su ejercicio parental eficaz en la vida cotidiana.

Desde esta perspectiva, han surgido en la primera década del siglo XXI otros elementos de estudio relacionados con la crianza: las habilidades parentales. Dicho concepto ha sido relacionado recientemente con Barudy (2010), quien inicialmente lo maneja como competencias parentales que engloban dos categorías: capacidades parentales fundamentales y habilidades parentales. Estas últimas hacen referencia al actuar de los padres en la vida cotidiana 
para estimular el desarrollo físico y psicológico de los menores. Cuando no existen estas habilidades en los padres, es porque en sus historias personales, familiares y sociales hay antecedentes de malos tratos infantiles, escasas o inadecuadas medidas de protección, institucionalización, pérdidas, entre otros.

Moral (2013) y Barudy (2010) enfatizan la tras-cendencia que tiene la preparación de ambos padres para llevar a cabo en la vida la crianza de los niños en la cotidianidad, ya que de esto depende su sano crecimiento. Si bien, hasta ahora parecieran escasos los estudios que se han ocupado de este tema, se puede enumerar su uso en dos ámbitos. Por un lado, en contextos judiciales donde su evaluación es útil para la determinación, por ejemplo, de la custodia y/o separación de los menores de sus progenitores (Reder, Duncan, \& Lucey, 2003). Por otro, en contextos de riesgo psicosocial, específicamente para identificar las capacidades de los padres para evitar y prevenir el maltrato infantil y generar programas de apoyo (Azar \& Cote, 2002).

En el ámbito pericial se encuentra la mayoría de las aportaciones. Las habilidades parentales son consideradas como un conjunto de conductas de los progenitores, necesarias para lograr la satisfactoria y sana crianza de sus hijos e hijas durante su desarrollo; parecen seguir una secuencia ordenada de interacciones en la comunicación parentofilial (Ramírez, 2003; Ramírez, Ibáñez, \& De Luis, 1994). Asimismo, Reder y Lucey (1995), las utilizan en este contexto y las asocian con diversas capacidades como funcionamiento personal (resiliencia, agencia personal), competencias asociadas al rol parental como tal, y competencias asociadas a la relación con el niño (Rodrigo, Martín, Cabrera, \& Máiquez, 2009).

Desde la perspectiva de familias en riesgo psicosocial, autores como Cabrera, Rincón y Vargas (1978), han agrupado las habilidades parentales en cinco categorías relacionadas con diversos ámbitos de la vida: 1. Habilidades educativas, 2. Agencia parental, 3. Autonomía y búsqueda de apoyo social, 4. Habilidades para la vida personal y de pareja y 5 . Organización doméstica.

La evaluación de las habilidades en ambos contextos (judicial y de riesgo psicosocial), van dirigidos a determinar si los padres cumplen con lo necesario para hacerse cargo de un menor. En estos enfoques sobre las habilidades parentales, el constructo se operacionaliza de manera clara y precisa; algunos autores incluyen elementos que otros modelos no contemplan.

Autores, como Azar y Cote (2002), han contemplado en un primer momento, el término de habilidades educativas, además, las sociocognitivas, el autocontrol, el manejo del estrés y las sociales, pues como se puede observar guardan estrecha relación y cierta generalidad para su evaluación en padres.

De igual forma, White (2005), engloba las habilidades parentales y las señala como el resultado de un ajuste entre las condiciones psicosociales en las que vive la familia, el escenario educativo que los padres o cuidadores han construido para realizar su tarea vital y las características del menor. Aunque no lo menciona de manera explícita, hace alusión a la interacción entre padres e hijos de acuerdo con el ciclo de la familia y el dinamismo propio que la caracteriza.

Un abordaje general de la evaluación de las competencias parentales lo realiza Barudy (2010), quien se basa en dos categorías: las capacidades parentales fundamentales, determinadas por factores biológicos y hereditarios, pero, al mismo tiempo, modulados por experiencias vitales e influenciados por la cultura y el entorno social. En esta categoría se comprende la capacidad de apegarse a los hijos, la empatía, los modelos de crianza, capacidad de participar en redes sociales y el uso de recursos comunitarios. Una segunda categoría la conforman las habilidades parentales, relacionadas con la plasticidad que deben tener los padres 
para adecuar su trato y sus respuestas a sus hijos de acuerdo a las necesidades madurativas, emocionales y de desarrollo de éstos.

Con dichos antecedentes, Rodrigo y Máiquez (2008), plantean de manera concreta habilidades parentales y las nombran como agencia parental, habilidades de autonomía y búsqueda de apoyo social, habilidades para la vida personal y de pareja, además de habilidades para la organización doméstica. Estas se constituyen como la base para una futura propuesta pionera con la intención de englobar indicadores que integren todas las capacidades de los padres. Los autores señalan que las habilidades parentales son un conjunto de capacidades para cuidar, proteger y educar a los hijos y contribuir a su desarrollo sano. El término competencias es un concepto integrador porque se refiere a la capacidad de las personas para generar y coordinar respuestas (afecto, cognición, comunicación y comportamiento) flexibles y adaptativas a corto y a largo plazo. Esto significa que las competencias parentales son multidimensionales (implican el funcionamiento integrado de la cognición, el afecto y el comportamiento), bidireccionales (propician el ajuste personal y social a los contextos y analizan lo que éstos proporcionan a las personas en su desarrollo), dinámicas (cambian de acuerdo al ciclo evolutivo del individuo) y contextuales (las tareas evolutivas se practican en contextos vitales $\mathrm{y}$, por otro lado, tales contextos ofrecen oportunidades para nuevos aprendizajes y prácticas).

Cabe mencionar que las habilidades parentales son requeridas para todas las etapas del desarrollo humano, con las particularidades de cada individuo, su etapa de desarrollo y el contexto en el cual se desenvuelve. Es importante tener presente que con la evaluación de las habilidades parentales no se pretende encasillar a los padres como perfectos/as o no, ya que no existen tales. Lo que se busca es identificar el grado de reconocimiento que cada progenitor o figura parental tiene sobre sus áreas fuertes y/o débiles en el ejercicio del rol, la actitud para buscar apoyo si éste fuera necesario, la existencia o no de balance entre las necesidades de la persona menor de edad y las posibilidades de los progenitores para resolverlas y, finalmente, la identificación de posibles factores de riesgo en la forma en que asumen el cuidado de los niños y niñas (Barudy, 2010).

Como se puede observar, los estudios sobre habilidades parentales se han realizado en mayor medida en países europeos; sin embargo, cabe destacar que en México existe una gran gama de investigaciones sobre crianza desde diferentes abordajes, como estilos, prácticas parentales y tareas de crianza (Flores-Galaz, Cortés-Ayala, \& Góngora-Coronado, 2003; Moral, 2013; Robles \& Oudhof, 2010; Palacios \& Andrade, 2008), las cuales se relacionan directamente con este constructo. Por ello, es interesante indagar cómo conciben muestras mexicanas las habilidades parentales a partir de las dimensiones ya propuestas en los antecedentes teóricos, identificar si estas mantienen su forma original, o si padres y madres de familia arrojan nuevas propuestas a la luz de la cultura mexicana. Con base en lo anterior surge esta investigación, cuyo objetivo es construir una escala válida y confiable que mida habilidades parentales en hombres y mujeres con hijos de entre 6 y 12 años de la ciudad de Toluca.

\section{Método}

\section{Participantes}

Se trabajó con 682 participantes, 341 hombres y 341 mujeres de la ciudad de Toluca. El único criterio de inclusión fue ser padre o madre de un niño o niña de entre 6 y 12 años, que se encontraran criándolo y que habitaran la misma vivienda. Para obtener la muestra se aplicó la regla de Reidl (2005), quien señala que cuando se construye una escala de medición, el total de los participantes se obtiene al multiplicar 
el número de reactivos existentes por cinco (para este caso $52 \times 5=260$ ). Sin embargo, cabe aclarar que debido a la alta participación de ambos padres, la muestra aumentó en $38.1 \%$, para conformar el total ya indicado.

\section{Instrumento}

La escala se construyó con base en las dimensiones planteadas por Rodrigo y Máiquez (2008), debido a la claridad conceptual con que las manejan. Los autores proponen cinco habilidades: educativas, agencia parental, autonomía y búsqueda de apoyo social, habilidades para la vida personal y de pareja, y para la organización doméstica. A partir de estas dimensiones se propusieron 63 reactivos tipo Likert con opciones de 1 (nunca) a 5 (siempre).

El diseño preliminar se sometió a validez de jueces y fue analizado por 5 expertos en el tema. Como resultado se obtuvieron 52 reactivos que finalmente se aplicaron en una muestra de 682 participantes; algunos términos del lenguaje fueron ajustados en esta fase. A todos los participantes se les habló sobre la confidencialidad de la información una vez que autorizaron su participación.

\section{Procedimiento}

El procedimiento que se llevó a cabo en esta investigación fue el siguiente:

a) Se realizó una extensa revisión teórica en diferentes medios como artículos científicos, libros especializados y publicaciones en revistas científicas. A partir de Rodrigo y Máiquez (2008), se crearon 5 dimensiones y 63 reactivos que constituyeron una escala preliminar tipo Likert, con opciones de respuesta que van de 1 (nunca) a 5 (siempre), como se muestra a continuación con ejemplos de algunos reactivos:

1. Educativa: "le digo cuánto lo quiero, lo apoyo cuando se le dificulta realizar su tarea".
2. Autopercepción parental: "me funciona la manera cómo educo a mi hijo, ser padre es muy difícil".

3. Vida personal: "tolero el mal comportamiento de mi hijo, dejo que mi pareja solucione los problemas".

4. Apoyo social: "acudo a las instituciones donde me orientan sobre cómo educar a mi hijo, le permito ir a las fiestas de sus amigos".

5. Organización doméstica: "mantengo la casa limpia y ordenada, faltan alimentos en casa".

a) La escala preliminar fue revisada por cinco expertos en el tema, quienes realizaron observaciones de redacción y coherencia, aprobaron 52 reactivos y eliminaron 9 de ellos por distintos motivos como falta de claridad o de una relación precisa con el constructo a medir.

b) Posteriormente, el instrumento fue piloteado, para ello se acudió a los hogares y centros de trabajo de los padres y madres de familia. Se les aplicó de manera individual, además, se les explicó el objetivo de la investigación y se enfatizó la confidencialidad de los datos con fines de investigación, asimismo, se les dio tiempo suficiente para contestarlo y se aclararon dudas cuando fue necesario. En el pilotaje no se modificaron los reactivos.

c) Finalmente, se procedió a la aplicación final de la escala en las mismas condiciones en que se llevó a cabo el pilotaje.

\section{Análisis de datos}

Para obtener la validez del instrumento se utilizó un análisis factorial de tipo exploratorio con el método de componentes principales de tipo ortogonal (rotación varimax). El criterio para obtener los factores se emitió de acuerdo con los autovalores mayores o iguales a 1. Se tomó en cuenta la claridad conceptual y el quiebre de la varianza, además, para considerar un fac- 
tor como tal, fue necesario que contara con un mínimo de tres reactivos positivos y cada uno de ellos una carga factorial de .40 hacia arriba. Para obtener la confiabilidad de la escala se obtuvo el alpha de Cronbach.

\section{Resultados}

De acuerdo con los resultados hallados, la estructura esperada no se aplicó ni a madres ni a padres. En el análisis factorial exploratorio, si bien las madres presentaron 5 factores, éstos no son similares a los planteados inicialmente, lo mismo sucedió con los padres, además de destacar que ellos presentaron 8 factores, es decir, 3 más que las mujeres.

Respecto a validez de la escala de habilidades parentales para madres, se encontraron 5 factores y 21 reactivos que explicaron $23.20 \%$ de la varianza, con una confiabilidad de alpha de Cronbach $=0.78$.

Asimismo, se obtuvo la confiabilidad de cada uno de los factores, siendo el más alto fue el factor 1 , afecto y apoyo ( $\alpha=0.77)$, y el más bajo fue el factor autoeficacia parental $(\alpha=0.50)$.

Tabla 1

Análisis factorial de la escala de habilidades parentales en madres

\begin{tabular}{lllllll}
\hline $\begin{array}{l}\text { Número } \\
\text { de factor }\end{array}$ & Nombre del factor & $\begin{array}{c}\text { Número de } \\
\text { reactivos }\end{array}$ & $\begin{array}{c}\text { Valores } \\
\text { Eigen }\end{array}$ & $\begin{array}{c}\text { Porcentaje de } \\
\text { la varianza }\end{array}$ & $\begin{array}{c}\text { Porcentaje de la } \\
\text { varianza acumulada }\end{array}$ & $\begin{array}{c}\text { Alpha de } \\
\text { Cronbach }\end{array}$ \\
\hline 1 & afecto & 7 & 7.41 & 6.42 & 6.42 & .77 \\
2 & acuerdo en la pareja & 3 & 2.51 & 4.68 & 11.10 & .73 \\
3 & limpieza y seguridad & 4 & 2.22 & 4.41 & 15.51 & .62 \\
4 & estimulación y desarrollo & 3 & 2.09 & 4.29 & 19.80 & .55 \\
5 & autoeficacia parental & 4 & 1.51 & 3.40 & 23.20 & .50 \\
\hline
\end{tabular}

A continuación, se muestran las cargas factoriales de cada uno de los reactivos y su ubicación dentro del factor. El factor afecto contó con 7 reactivos. Limpieza y seguridad y autoeficacia parental con 4. Finalmente acuerdos con la pareja y estimulación y desarrollo con 3 reactivos.

En la validez de la escala de habilidades parentales para padres, se obtuvieron 8 factores distribuidos en 29 reactivos, que explicaron $38.75 \%$ de la varianza y una confiabilidad de alpha de Cronbach de .85.
La confiabilidad de cada uno de los factores, arrojó que el más alto fue el factor 1 salud y valores $(\alpha=.73)$ y el más bajo fue el factor 8 aceptación del menor $(\alpha=.53)$.

La carga factorial y la distribución de los reactivos por factor fue de la siguiente manera: los factores valores y estimulación y desarrollo contaron con 5 reactivos; acuerdos con la pareja con 4; solución de problemas, afecto, autoeficacia parental, limpieza y seguridad y aceptación del menor, con 3 reactivos. 
Tabla 2

Reactivos de la escala de habilidades parentales en madres de familia

\begin{tabular}{|c|c|c|c|c|c|}
\hline $\begin{array}{c}\text { Factor } 1 \\
\text { Afecto y apoyo }\end{array}$ & & & & & \\
\hline Lo felicito por sus logros & .72 & & & & \\
\hline Le digo cuánto lo quiero & .69 & & & & \\
\hline Le pregunto cómo se siente & .68 & & & & \\
\hline Abrazo a mi hijo (a) & .62 & & & & \\
\hline Lo apoyo cuando se le dificulta realizar su tarea & .48 & & & & \\
\hline Halago a mi hijo por sus cualidades & .44 & & & & \\
\hline Le enseño el respeto hacia los demás & .40 & & & & \\
\hline \multicolumn{6}{|l|}{$\begin{array}{c}\text { Factor } 2 \\
\text { Acuerdo en la pareja }\end{array}$} \\
\hline Cuando tomo una decisión respecto a mi hijo mi pareja me respalda & & .82 & & & \\
\hline Mi pareja y yo concordamos en cuáles son los límites y reglas en el hogar & & .78 & & & \\
\hline Respetamos los acuerdos sobre la educación de mis hijos & & .61 & & & \\
\hline \multicolumn{6}{|l|}{$\begin{array}{c}\text { Factor } 3 \\
\text { Limpieza y seguridad }\end{array}$} \\
\hline Recogemos las cosas después de usarlas & & & .67 & & \\
\hline Conozco cómo educar a mis hijos & & & .63 & & \\
\hline Mantengo la casa limpia y ordenada & & & .61 & & \\
\hline Ante un problema actúo de manera correcta en el momento oportuno & & & .56 & & \\
\hline \multicolumn{6}{|l|}{$\begin{array}{c}\text { Factor } 4 \\
\text { Estimulación y desarrollo }\end{array}$} \\
\hline Le compro libros diferentes a los de la escuela & & & & .65 & \\
\hline Compro juguetes que estimulen su desarrollo & & & & .59 & \\
\hline Lo llevo a lugares para que conozca nuevos amigos & & & & .58 & \\
\hline \multicolumn{6}{|l|}{$\begin{array}{c}\text { Factor } 5 \\
\text { Autoeficacia parental }\end{array}$} \\
\hline Como padre pongo el ejemplo de cómo debe actuar & & & & & .69 \\
\hline Tengo claras mis funciones como padre o madre. & & & & & .42 \\
\hline Le enseño a cuidar el medio ambiente & & & & & .41 \\
\hline Planeo las cosas para lograr lo que quiero & & & & & .40 \\
\hline
\end{tabular}

\section{Tabla 3}

Análisis factorial de la escala de habilidades parentales en los hombres

\begin{tabular}{clccccc}
\hline $\begin{array}{c}\text { Número } \\
\text { de factor }\end{array}$ & $\begin{array}{l}\text { Nombre del } \\
\text { factor }\end{array}$ & Número de reactivos & Valores eigen & $\begin{array}{c}\text { Porcentaje de } \\
\text { la varianza }\end{array}$ & $\begin{array}{c}\text { Porcentaje de } \\
\text { la varianza } \\
\text { acumulada }\end{array}$ & $\begin{array}{c}\text { Alpha de } \\
\text { Crobach }\end{array}$ \\
\hline 1 & valores & 5 & 9.01 & 5.86 & 5.86 & .73 \\
2 & $\begin{array}{l}\text { estimulación } \\
\text { y desarrollo }\end{array}$ & 5 & 2.79 & 5.78 & 11.64 & .71 \\
3 & $\begin{array}{l}\text { acuerdos en } \\
\text { la pareja }\end{array}$ & 4 & 2.50 & 5.62 & 17.26 & .75 \\
4 & $\begin{array}{l}\text { solución de } \\
\text { problemas }\end{array}$ & 3 & 1.99 & 4.50 & 21.76 & .58 \\
5 & $\begin{array}{l}\text { afecto } \\
\text { autoeficacia }\end{array}$ & 3 & 1.70 & 4.45 & 26.21 & .67 \\
6 & $\begin{array}{l}\text { parental } \\
\text { limpieza y }\end{array}$ & 3 & 1.52 & 3.84 & 30.05 & .61 \\
7 & $\begin{array}{l}\text { seguridad } \\
\text { aceptación }\end{array}$ & 3 & 1.46 & 3.73 & 33.78 & .58 \\
8 & del menor & 3.41 & 3.50 & 37.28 & .53 \\
\hline
\end{tabular}




\section{Tabla 4}

\section{Reactivos de la escala de habilidades parentales en padres}

\begin{tabular}{|c|c|c|}
\hline $\begin{array}{l}\text { Factor } 1 \\
\text { Valores }\end{array}$ & & \\
\hline Le enseño el respeto hacia los demás & .62 & \\
\hline Como padre pongo el ejemplo de cómo debe actuar & .58 & \\
\hline Planeo las cosas para lograr lo que quiero & .57 & \\
\hline Conozco la pirámide nutricional & .49 & \\
\hline Le enseño a cuidar el medio ambiente & .49 & \\
\hline \multicolumn{3}{|l|}{$\begin{array}{l}\text { Factor } 2 \\
\text { Estimulación y desarrollo }\end{array}$} \\
\hline Le compro libros diferentes a los de la escuela & & .72 \\
\hline $\begin{array}{l}\text { Lo llevo a realizar actividades después de clases como deporte, música } \\
\text { o baile }\end{array}$ & & .65 \\
\hline Lo llevo a lugares para que conozca nuevos amigos & & .63 \\
\hline Compro juguetes que estimulen su desarrollo & & .55 \\
\hline En casa hay frutas y verduras & & .44 \\
\hline
\end{tabular}

\section{Factor 3}

Acuerdos en la pareja

Cuando tomo una decisión respecto a mi hijo mi pareja me respalda $\quad .73$

Respetamos los acuerdos sobre la educación de mis hijos $\quad .70$

Mi pareja y yo concordamos cuáles son los límites y reglas en el hogar

$\begin{array}{ll}\text { Tengo claras mis funciones como padre o madre } & .73\end{array}$

Factor 4

Solución de problemas

Ante un problema no sé cómo actuar

Cuando mi hijo me desobedece no sé cómo actuar $\quad .69$

Actuó de forma impulsiva ante un problema

\section{Factor 5}

Afecto

Le digo cuánto lo quiero

Factor 6

Autoeficacia Parental

Conozco cómo educar a mis hijos

Me funciona la manera como educo a mi hijo

Ante un problema actúo de manera correcta en el momento oportuno

Mantengo la casa limpia y ordenada

Recogemos las cosas después de usarlas

Mantengo la casa libre de riesgos

Factor 8 Aceptación del menor

Estoy contento con lo que ha aprendido mi hijo 


\section{Discusión}

De acuerdo con los resultados, padres y madres de familia consideran que poseen habilidades como afecto, acuerdos en la pareja, limpieza y seguridad, estimulación y desarrollo y autoeficacia parental. Cabe destacar que los varones obtuvieron tres factores más que las madres: valores, solución de problemas y aceptación de las características del menor. Esto corrobora la gran trascendencia que guarda la familia, pues es sin duda el lugar donde los niños tienen sus primeras interacciones con el mundo. Madres y padres invierten en la crianza de sus hijos en busca de las mejores técnicas para lograrlo (Frías, Fraijo, \& Tapia, 2012).

Entre las habilidades registradas en ambos padres se puede destacar el afecto, que tiene relación con la expresividad, el mostrar cariño, ser emotivos. En el contexto social mexicano se inculca en las mujeres ser más expresivas emocionalmente que los varones, sin embargo, el hallazgo revela que ambos le dan la misma importancia a este aspecto. La relevancia de la afectividad, radica en la susceptibilidad del ser humano frente a distintas alteraciones del mundo real o simbólico, por lo general, se produce a través de un proceso interactivo (Brett, 2010). En lo que respecta a los acuerdos en la pareja, Reyes, Garrido, Ortega y Torres (2007) hacen énfasis en que son básicos en la relación de la pareja y ello favorece su éxito como familia y evita el divorcio. La negociación, el diálogo y la comprensión contribuyen al desarrollo sano de los hijos.

Limpieza y seguridad conforman otro factor común en madres y padres, de hecho son parte de la supervivencia de los menores. Reyes, et al. (2007) consideran que los hábitos de higiene personal son básicos para la salud y la estabilidad emocional, así como el desarrollo sano de los menores. Los adultos son quienes se disponen a enseñarlos debido a que ellos ya los han desarrollado; su papel es representar modelos que los menores imitarán.

El factor estimulación y desarrollo es fundamental en los menores, pues los fortalece como individuos y previene problemas en cualquiera de las esferas del desarrollo biopsicosocial. Esta idea es apoyada por González y Tovar (1994), al señalar que la estimulación de habilidades especificas en los menores regula su proceso vital y contribuye a hacerlos autosuficientes.

Cuando ambos padres consideran que están realizando la crianza de manera adecuada, tienen a la mano un elemento que les permite continuar en la vida cotidiana o bien, parar y, de ser necesario, modificar aquello que consideran disfuncional para sus hijos. Dicha situación guarda relación con la autoeficacia parental que Farkas-Klein (2008) conceptualiza como las creencias personales sobre las propias capacidades para lograr ser un buen padre, su importancia radica en qué tanto se puede estar satisfecho y capaz de hacer lo necesario para perseverar y lograr una tarea determinada, lo que no significa ser un padre bueno o perfecto, más bien implica tener claro que se pueden cometer errores y aprender de ellos.

Respecto a la existencia de otros factores en la muestra de hombres, valores representa el primero de ellos: se centra en enseñar el comportamiento socialmente esperado a los hijos, ya que en el contexto en el que se vive, los padres juegan, además, el rol de figura de autoridad, en quien recae la corrección del mal comportamiento. Garay et al. (2009), hacen alusión a los valores como constructos socioculturales abstractos de los que dependen los intereses específicos de cada individuo. Los valores son las metas deseables y sirven como principios que guían la vida de los seres humanos, de ahí la importancia que toma para los padres como habilidad parental. Lo mismo sucede con el factor solución de problemas. Los padres tratan de desarrollar en sus menores esta habilidad, ya que entre los reactivos de la escala se puede leer que consideran la experimentación como la mejor forma de aprender y guiar la resolución de conflictos e intrínseca al desempeño social (Cabrera, Rincon, \& Vargas, 1978).

Otro factor encontrado en varones fue el de aceptación de las características del menor, que se refiere a aceptar a los niños de acuerdo 
a su edad y nivel de desarrollo. El error común en los padres es ignorar qué deben hacer con su hijo y pedirles repertorios mayores a los que pueden realizar. Cuando los padres se hacen partícipes en el proceso de aprendizaje, conocen mejor las características propias de sus hijos y lo que se espera realicen desde las etapas tempranas de su vida, esta perspectiva genera un beneficio claro para éste. Si los padres reciben información sobre las etapas del desarrollo, se pueden hacer conscientes de lo que los menores pueden realizar en cada etapa de desarrollo según la edad y la madurez (Rodríguez, 2004).

De alguna manera, estos hallazgos concuerdan con otros estudios como el de Frías, et al. (2012) quienes indagaron la percepción del estilo de crianza de padres. Asimismo, en un estudio de Moral (2013), se describieron las prácticas parentales y la definición que cada padre tenía de sí mismo. Los resultados arrojaron de manera general que los padres y madres tienen una buena percepción de su desempeño en la crianza, al igual que lo referido en este estudio.

Si bien estos datos establecen similitudes con otras investigaciones, una limitante es que estos estudios no se han analizado a luz de la perspectiva de género, es decir, resulta imprescindible indagar el significado real que hombres y mujeres le dan a las habilidades parentales desde su subjetividad.

Asimismo, es preciso revisar si en el hogar y en la vida de madres y padres de familia, la medición de este constructo arroja datos similares o es diferente a lo hallado en esta investigación. Para tal fin, se considera necesario abordarlo más bien desde los métodos cualitativos.

Finalmente, se precisa que a futuro se trabaje con muestras más grandes de madres y padres de familia que permitan realizar un análisis factorial de tipo confirmatorio.
Azar, S., \& Cote, L. (2002). Sociocultural issues in the evaluation of the needs of children in custody decision making: What do our current frameworks for evaluating parenting practices have to offer? International Journal of Law Psychiatry, 25(3), 193-217.

Barudy, J. (2010). Los buenos tratos y la resiliencia infantil en la prevención de los trastornos del comportamiento. Madrid: Gedisa.

Brett, G. (2010). Historia de la psicología. Paidós: México.

Cabrera, H., Rincón, P., \& Vargas, A. (1978). Estilos de solución de conflictos. Revista Latinoamericana de Psicología, 10(1), 53-69.

Farkas-Klein, C. (2008). Escala de evaluación parental (EEP): desarrollo, propiedades psicométricas y aplicaciones. Pontificia Universidad Católica, 7(2), 457-467.

Flores-Galaz, M. M., Cortés-Ayala, M. L., \& Góngora-Coronado, E. A. (2003). Estilos de crianza: una aproximación a su identificación en familias de Yucatán. Educación y Ciencia (México), 7(14), 31-42.

Frías, M., Fraijo, B., \& Tapia, C. (2012). Prácticas de crianza y desarrollo emocional y afectivo de los niños. PSICUMEX, (4), 30-41.

Garay, J., Díaz-Loving, R., Cárdenas, M., Limón, B., Lozano I., Rocha, T., \& Zacarías, M. (2009). Intereses y valores en jóvenes mexicanos. Enseñanza e Investigación en Psicología, 14(2), 295-309.

González, F., \& Tovar, G. (1994). Un análisis sobre la supuesta participación masculina en el trabajo doméstico. Tesis de licenciatura, Universidad Nacional Autónoma México: UNAM.

Moral, J. (2013). Prácticas parentales y percepción del rol en padres adolescentes. Revista Mexicana de Investigación en Psicología, 5(1), 6-19.

Musitu, G., \& Cava, M. (2001). La Familia y la Educación. Barcelona: Octaedro.

Musitu, G., \& García, G. (2001). Escala de Socialización Parental en la adolescencia. Madrid: TEA Ediciones.

Palacios, D., \& Andrade, P. (2008). Influencia de las prácticas parentales en las conducta problema en adolescentes. Ciencias Sociales y Humanidades, 7(7). 7-18.

Ramírez, G. M. (2003). Cuando los padres se separan. Madrid: Biblioteca Nueva.

Ramírez, M., Ibáñez, V., \& De Luis, P. (1994). Percepciones Parentales en niños de familias separadas. Anuario de Psicología Jurídica, 14(1), 25-44.

Reder, P., \& Lucey, C. (1995). Assessment of parenting: Psychiatric and psychological contributions. London: Routledge.

Reder, P., Duncan, S., \& Lucey, C. (2003). Studies in the assessment of parenting. Nueva York: Routledge.

Reidl, L. (2005). Perspectiva de la investigación en psicología social. Revista de Psicología Social y Personalidad, 21(1), 50-62.

Reyes, L., Garrido, A., Ortega, S., \& Torres, L. (2007). La vida en pareja: un asunto a negociar. Revista de Enseñanza e Investigación en Psicología, 12(2), 385-396.

Robles, E., \& Oudhof, H. (2010). Validación del cuestionario de tareas de crianza en una muestra de mujeres mexicanas. Pensamiento Psicológico, 7(14), 73-80.

Rodrigo, M., \& Máiquez, C., (2008). Las Competencias Parentales en Contextos de Riesgo Psicosocial. Intervención Psicosocial, 18(2), 113-120.

Rodrigo, M., Martín, J. C., Cabrera, E., \& Máiquez. C. (2009). Las Competencias Parentales en Contextos de Riesgo Psicosocial. Psychosocial Intervention, 18 (2), 113-120.

Rodríguez, P. (2004). Desarrollo Psicosocial de los niños y las niñas. Bogotá: UNICEF.

White, A. (2005). Assessment of parenting capacity. Literature review. Centre for Parenting and Research. Australia: Department of Community Services. 